\title{
COMPATIBILIZAÇÃO DE REFERENCIAIS DE COORDENADAS E VELOCIDADES COM ESTIMATIVA DE PRECISÃO
}

\section{Compatibilization of coordinate reference systems and velocities with estimate of precision}

\author{
Alessandro Salles Carvalho ${ }^{1,2}$ \\ William Rodrigo Dal Poz ${ }^{2}$ \\ Ana Paula Camargo Larocca ${ }^{3}$ \\ 1 Universidade Federal de Juiz de Fora, Juiz de Fora - MG, Brasil, email: \\ alessandro.carvalho@ufjf.edu.br. \\ 2Universidade Federal de Viçosa, Viçosa -MG, Brasil, email: william.dalpoz@ufv.br \\ 3Universidade de São Paulo/Escola de Engenharia de São Carlos, São Carlos -SP, Brasil, email: \\ larocca.ana@usp.br
}

\section{Resumo:}

As transformações de coordenadas e velocidades entre as realizações do ITRS (International Terrestrial Reference System), bem como a atualização de coordenadas, tornar-se-ão tarefas rotineiras em levantamentos geodésicos devido ao emprego de sistemas de referência dinâmicos e ao movimento das placas tectônicas. Neste estudo foram realizadas compatibilizações de sistemas de referência (ITRF2000, ITRF2005 e ITRF2008) das coordenadas e velocidades com suas respectivas estimativas de precisão, via propagação de variância, de 11 estações distribuídas nas placas tectônicas Norte e Sul Americanas e da Eurásia. Foi verificado com base nas coordenadas nos ITRF2008, época 2005 e ITRF2005, época 2000, obtidas pelo IERS (International Earth Rotation and Reference System Service), que 54,55\% das discrepâncias planimétricas são centimétricas e 45,45\% decimétricas. Comparando as coordenadas no ITRF2008, época 2005,0 e no ITRF2000, época 1997,0 verificou-se discrepâncias planimétricas da ordem do decímetro para todas as estações. Confrontando as coordenadas ITRF2000, época 1997,0 obtidas pelo IERS com as calculadas com base nas transformações entre sistemas de referência e atualizações verifica-se discrepâncias planimétricas da ordem do milímetro em 72,73\% dos casos e da ordem do centímetro em 27,27\%. As análises realizadas confirmam a necessidade de atualizar e compatibilizar o referencial das coordenadas e velocidades para aumentar a acurácia do posicionamento.

Palavras-chave: Sistemas de Referência; Propagação de variância; Parâmetros de transformação; Atualização de coordenadas.

\section{Abstract:}

The transformation of coordinates and velocities between realizations of ITRS (International Terrestrial Reference System) and update of coordinates should become routine task in geodetic surveys due to the use of dynamic reference systems and the movement of tectonic plates. In this study, it was performed the compatibilization of the reference frames (ITRF2000, ITRF2005, and ITRF2008) of coordinates and velocities with estimation of precision considering propagation of 
variance of 11 stations distributed in the North American, South American, and Eurasian plate tectonics. Based on the coordinates in the ITRF2008, epoch 2005 and ITRF2005, epoch 2000.0, obtained by IERS (International Earth Rotation and Reference System Service), it was verified that $54.55 \%$ of the horizontal discrepancies are on the order of centimeters and $45.45 \%$ are on the order of decimeters. By comparing the coordinates in the ITRF2008, epoch 2005.0 and ITRF2000, epoch 1997.0, it was found horizontal discrepancies on the order of decimeters for all stations. However, comparing the coordinates of ITRF2000, epoch 1997.0, obtained by IERS, with the computed coordinates based on transformations between reference frames and updates, it was found horizontal discrepancies on the order of millimeters in $72.73 \%$ of the sites and on the order of centimeters in $27.27 \%$. The analyzes confirm that it is necessary to update and make the compatibilization of coordinate reference systems and velocities in order to increase the accuracy in the positioning.

Keywords: Reference Frame; Propagation of Variances; Transformation Parameters; Update of coordinates.

\section{Introdução}

No posicionamento geodésico com o uso do GPS (Global Positioning System) realizado com base na utilização de métodos relativos estáticos de alta precisão ou por ponto preciso, pós-processado ou em tempo real, são empregadas efemérides precisas as quais são vinculadas à época da sessão do rastreio e ao referencial realizado pelo IGS (International GNSS Service).

O IGS altera regularmente o sistema de referência o qual é utilizado para vincular seus produtos, entre eles, efemérides precisas, relacionadas às realizações ITRF (International Terrestrial Reference Frame) do ITRS (International Terrestrial Reference System) (IGS 2014). Desde 07/10/2012 (semana GPS 1709) até o presente, o sistema de referência utilizado pelo IGS é denominado de IGb08, o qual é alinhado ao ITRF2008 (Rebischung 2012).

Na transformação de coordenadas vinculadas ao ITRF2008, em uma época específica para os ITRF2005 e ITRF2000 em outras épocas, é necessário conhecer os parâmetros que os relacionam e as velocidades das estações posicionadas na placa tectônica (Weston and Soler 2012); (SIRGAS 2014).

A mudança de sistema de referência, como por exemplo, do ITRF2008 para realizações anteriores do ITRS, pode ser feita por meio da transformação de Helmert com utilização de 14 parâmetros de transformação (três translações, três rotações, um fator de escala e respectivas variações temporais (rates)) (Soler and Snay 2004).

A atualização de coordenadas é o processo no qual são utilizadas as velocidades das estações para que sejam consideradas sua variação espaço-temporal. As coordenadas são expressas em função do tempo para que reflitam a realidade do movimento da crosta associado com a tectônica de placas, subsidência ou soerguimento da superfície terrestre, atividades vulcânicas, entre outros (Soler and Snay 2004). Na área de abrangência do SIRGAS (Sistema de Referência Geocêntrico para as Américas) recomenda-se a utilização do modelo VEMOS2009 (Velocity Model for SIRGAS) (Drewes and Heidbach 2012); (Sánchez et al. 2013); (SIRGAS 2014).

Pimenta et al. (2002) realizaram experimentos utilizando nove estações em três placas tectônicas os quais transformaram coordenadas do ITRF2000 para os ITRF93 e ITRF97 e as compararam 
com as soluções disponibilizadas pelo IERS. As velocidades das estações foram obtidas a partir do modelo de movimento de placas tectônicas NNR-NUVEL 1A (No Net Rotation-Northwesterm University VELocity model 1A) não sendo contemplado a propagação de variância na atualização e transformação entre sistemas de referência para estimativa das precisões das coordenadas transformadas.

Soler and Marshall (2002) desenvolveram um estudo de caso contemplando a propagação de variância na atualização e transformação de sistemas de referência das coordenadas e velocidades do ITRF2000 para o ITRF97 na época 1997,0. Os resultados sugeriram que para obtenção da máxima acurácia nesses procedimentos, deve-se ter disponível a Matriz Variância-Covariância (MVC) completa dos 14 parâmetros de transformação.

A Transformação do referencial das coordenadas das estações da rede passiva mexicana do ITRF92, época 1988,0 para ITRF2000, época 2004,0 com base nos parâmetros disponibilizados pelo IERS (International Earth Rotation and Reference System Service) e nos modelos globais de movimento de placas tectônicas tais como ITRF2000 plate motion, NNR-NUVEL 1A e APKIM (Actual Kinematic and Crustal Deformation Model) foi realizado por Franco and Moreno (2006). Estes autores propuseram um modelo que permite quantificar a influência da mudança de referencial e do deslocamento da placa tectônica na determinação das coordenadas das estações.

Haasdyk and Janssen (2011) realizaram atualizações e mudanças de referenciais do GDA94 (Geocentric Datum of Australia) para ITRF considerando-se propagação de variância com intuito de demonstrar o efeito das transformações na qualidade dos dados (coordenadas transformadas).

Prol et al. (2014) desenvolveram um aplicativo, disponível na web, denominado TREVel (Transformação entre Referenciais e cálculo de Velocidades) que permite a transformação entre coordenadas referenciadas ao ITRF e SIRGAS2000. Porém, nota-se que o mesmo não permite estimativa de precisão das coordenadas transformadas.

Neste trabalho realizou-se a transformação de referencial das coordenadas e velocidades do ITRF2008, época 2005,0 para os ITRF2005, época 2000,0 e ITRF2000, época 1997,0 com estimativa de suas precisões. Nesse contexto, foram analisadas as discrepâncias posicionais em termos de coordenadas geodésicas (latitude, longitude a altitude elipsoidal) de 11 estações no ITRF2000, época 1997,0 e ITRF2005, época 2000,0 calculadas a partir do ITRF2008, época 2005,0 em relação às soluções fornecidas pelo IERS. As discrepâncias planimétricas e tridimensionais foram quantificadas a partir da comparação das coordenadas obtidas com e sem a consideração dos procedimentos de atualização e transformação de referencial das coordenadas e velocidades das estações.

\section{Transformação e atualização de coordenadas entre sistemas de referência}

A transformação de Helmert é utilizada para relacionar dois sistemas de referência com base nos 14 parâmetros de transformação (Petit and Luzum 2010). As velocidades dos parâmetros servem para atualizá-los de sua época de referência $\left(\mathrm{t}_{\mathrm{k}}\right)$ para uma época de interesse, a qual normalmente coincide com a época das coordenadas $\left(\mathrm{t}_{0}\right)$ no sistema de referência origem. Detalhes da transformação de referencial das coordenadas e velocidades podem ser encontrados em (Altamimi, Collilieux, and Métivier 2012); (Petit and Luzum 2010). 
O modelo matemático utilizado para transformação das coordenadas no sistema referência de origem (o) para o sistema de referência destino (d) pode ser dado por meio da equação matricial (1) (ITRF 2014):

$$
\left[\begin{array}{c}
\mathrm{X} \\
\mathrm{Y} \\
\mathrm{Z}
\end{array}\right]_{\mathrm{d}}=\left[\begin{array}{c}
\mathrm{X} \\
\mathrm{Y} \\
\mathrm{Z}
\end{array}\right]_{0}+\left[\begin{array}{c}
\mathrm{T}_{\mathrm{X}} \\
\mathrm{T}_{\mathrm{Y}} \\
\mathrm{T}_{\mathrm{Z}}
\end{array}\right]+\left[\begin{array}{ccc}
\mathrm{D} & -\mathrm{R}_{\mathrm{Z}} & \mathrm{R}_{\mathrm{Y}} \\
\mathrm{R}_{\mathrm{Z}} & \mathrm{D} & -\mathrm{R}_{\mathrm{X}} \\
-\mathrm{R}_{\mathrm{Y}} & \mathrm{R}_{\mathrm{X}} & \mathrm{D}
\end{array}\right]\left[\begin{array}{c}
\mathrm{X} \\
\mathrm{Y} \\
\mathrm{Z}
\end{array}\right]_{0}
$$

Em que:

$\left[\begin{array}{lll}\mathrm{X} & \mathrm{Y} & \mathrm{Z}\end{array}\right]_{\mathrm{d}}^{\mathrm{T}}$ :vetor das coordenadas cartesianas geocêntricas no sistema de referência destino;

$\left[\begin{array}{lll}\mathrm{X} & \mathrm{Y} & \mathrm{Z}\end{array}\right]_{0}^{\mathrm{T}}$ :vetor das coordenadas cartesianas geocêntricas no sistema de referência origem;

$\left[\begin{array}{lll}T_{\mathrm{X}} & \mathrm{T}_{\mathrm{Y}} & \mathrm{T}_{\mathrm{Z}}\end{array}\right]^{\mathrm{T}}$ :vetor das translações;

$\mathrm{R}_{\mathrm{X}}, \mathrm{R}_{\mathrm{Y}}, \mathrm{R}_{\mathrm{Z}}$ : rotações diferenciais em torno dos eixos $\mathrm{X}, \mathrm{Y}, \mathrm{Z}$, respectivamente, dadas em radianos; $\mathrm{e}$

D: fator diferença de escala, adimensional.

Na utilização da Equação 1 é necessário que os sete parâmetros de transformação estejam na mesma época de referência das coordenadas das estações no sistema origem. A atualização dos parâmetros pode ser realizada por meio da Equação 2 (Soler and Snay 2004); (ITRF 2014).

Em que:

$$
\mathbf{p}\left(\mathrm{t}_{0}\right)=\mathbf{p}\left(\mathrm{t}_{\mathrm{k}}\right)+\dot{\mathbf{p}} \cdot \Delta \mathrm{t}
$$

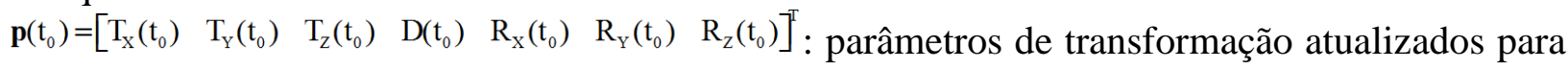
a época $\left({ }^{t_{0}}\right)$ de referência das coordenadas no sistema origem;

$\mathbf{p}\left(\mathrm{t}_{\mathrm{k}}\right)=\left[\begin{array}{lllllll}\mathrm{T}_{\mathrm{X}}\left(\mathrm{t}_{\mathrm{k}}\right) & \mathrm{T}_{\mathrm{Y}}\left(\mathrm{t}_{\mathrm{k}}\right) & \mathrm{T}_{\mathrm{Z}}\left(\mathrm{t}_{\mathrm{k}}\right) & \mathrm{D}\left(\mathrm{t}_{\mathrm{k}}\right) & \mathrm{R}_{\mathrm{X}}\left(\mathrm{t}_{\mathrm{k}}\right) & \mathrm{R}_{\mathrm{Y}}\left(\mathrm{t}_{\mathrm{k}}\right) & \mathrm{R}_{\mathrm{Z}}\left(\mathrm{t}_{\mathrm{k}}\right)\end{array}\right]^{\mathrm{T}}$ : vetor dos parâmetros de transformação disponibilizados na época $\left({ }^{t_{k}}\right)$;

$\dot{\mathbf{p}}=\left[\begin{array}{lllllll}\dot{\mathrm{T}}_{\mathrm{X}} & \dot{\mathrm{T}}_{\mathrm{Y}} & \dot{\mathrm{T}}_{\mathrm{Z}} & \dot{\mathrm{D}}^{\mathrm{D}} & \dot{\mathrm{R}}_{\mathrm{X}} & \dot{\mathrm{R}}_{\mathrm{Y}} & \dot{\mathrm{R}}_{\mathrm{Z}}\end{array}\right]^{\mathrm{T}}$ : vetor das variações temporais (rates) dos parâmetros de transformação;

$\Delta t=\left(t_{0}-t_{k}\right)$ : intervalo de tempo decorrido entre a época de referência dos parâmetros e a época de interesse;

$\mathrm{t}_{0}$ : época para qual serão atualizados os parâmetros; $\mathrm{e}$

$\mathrm{t}_{\mathrm{k}}$ : época de referência dos parâmetros.

A época de interesse para determinação dos parâmetros deverá ser igual à época $\left(\mathrm{t}_{0}\right)$ das coordenadas no sistema de referência origem e por isso é necessária a atualização dos parâmetros.

As velocidades das estações deverão estar no mesmo referencial das coordenadas para que estas possam ser atualizadas da época $\left(\mathrm{t}_{0}\right)$ para a época de interesse $(\mathrm{t})$. A mudança de referencial das velocidades pode ser efetuada com o emprego da Equação 3 (Altamimi, Collilieux, and Métivier 2012): 


$$
\left[\begin{array}{c}
\mathrm{V}_{\mathrm{X}} \\
\mathrm{V}_{\mathrm{Y}} \\
\mathrm{V}_{\mathrm{Z}}
\end{array}\right]_{\mathrm{d}}=\left[\begin{array}{c}
\mathrm{V}_{\mathrm{X}} \\
\mathrm{V}_{\mathrm{Y}} \\
\mathrm{V}_{\mathrm{Z}}
\end{array}\right]_{0}+\left[\begin{array}{c}
\dot{\mathrm{T}}_{\mathrm{X}} \\
\dot{\mathrm{T}}_{\mathrm{Y}} \\
\dot{\mathrm{T}}_{\mathrm{Z}}
\end{array}\right]+\dot{\mathrm{D}}+\left[\begin{array}{ccc}
\dot{\mathrm{D}} & -\dot{\mathrm{R}}_{\mathrm{Z}} & \dot{\mathrm{R}}_{\mathrm{Y}} \\
\dot{\mathrm{R}}_{\mathrm{Z}} & \dot{\mathrm{D}} & -\dot{\mathrm{R}}_{\mathrm{X}} \\
-\dot{\mathrm{R}}_{\mathrm{Y}} & \dot{\mathrm{R}}_{\mathrm{X}} & \dot{\mathrm{D}}
\end{array}\right]\left[\begin{array}{c}
\mathrm{X} \\
\mathrm{Y} \\
\mathrm{Z}
\end{array}\right]_{0}
$$

Em que:

$\left[\begin{array}{lll}\mathrm{v}_{\mathrm{X}} & \mathrm{V}_{\mathrm{Y}} & \mathrm{V}_{\mathrm{Z}}\end{array}\right]_{\circ}^{\mathrm{T}}$ : vetor das velocidades das estações no sistema de referência origem; e

$\left[\begin{array}{lll}\mathrm{V}_{\mathrm{X}} & \mathrm{V}_{\mathrm{Y}} & \mathrm{V}_{\mathrm{Z}}\end{array}\right]_{\mathrm{d}}^{\mathrm{T}}$ : vetor das velocidades lineares das estações no sistema de referência destino, dadas em metros por ano.

Nas Tabelas 1 e 2 estão disponíveis os parâmetros, variações temporais e respectivas precisões para transformação do referencial das coordenadas e velocidades do ITRF2008 para ITRF2005 e do ITRF2005 para ITRF2000.

Tabela 1: Parâmetros de transformação do ITRF2008 para ITRF2005 - época 2005,0.

\begin{tabular}{|c|c|c|c|c|c|c|c|}
\hline & $\mathrm{T}_{\mathrm{X}}(\mathrm{m})$ & $\mathrm{T}_{\mathrm{Y}}(\mathrm{m})$ & $\mathbf{T}_{\mathrm{Z}}(\mathbf{m})$ & $\mathbf{D}(\mathbf{p p b})$ & $\mathbf{R}_{\mathbf{X}}$ (mas) & $\mathbf{R}_{\mathbf{Y}}$ (mas) & $\mathbf{R}_{\mathrm{Z}}$ (mas) \\
\hline $\begin{array}{c}\text { Parâmetros e } \\
\text { precisões }\end{array}$ & $-0,0005$ & $-0,0009$ & $-0,0047$ & 0,94 & 0,000 & 0,000 & 0,000 \\
\hline \pm & 0,0002 & 0,0002 & 0,0002 & 0,03 & 0,008 & 0,008 & 0,008 \\
\hline \multirow[t]{2}{*}{$\begin{array}{l}\text { Variações temporais } \\
\text { e precisões }\end{array}$} & $\dot{\mathrm{T}}_{\mathrm{X}}(\mathrm{m} / \mathbf{a n o})$ & $\dot{\mathrm{T}}_{\mathrm{Y}}(\mathrm{m} / \mathbf{a n o})$ & $\dot{\mathrm{T}}_{\mathrm{Z}}(\mathrm{m} / \mathbf{a n o})$ & $\begin{array}{c}\dot{\mathbf{D}} \text { (ppb/ } \\
\text { ano) }\end{array}$ & $\dot{\mathbf{R}}_{\mathbf{X}}(\mathbf{m a s} / \mathbf{a n o})$ & $\dot{\mathbf{R}}_{\mathbf{Y}}(\mathbf{m a s} / \mathbf{a n o})$ & $\dot{\mathbf{R}}_{\mathrm{Z}}(\mathbf{m a s} / \mathbf{a n o})$ \\
\hline & 0,0003 & 0,000 & 0,000 & 0,00 & 0,000 & 0,000 & 0,000 \\
\hline \pm & 0,0002 & 0,0002 & 0,0002 & 0,03 & 0,008 & 0,008 & 0,008 \\
\hline
\end{tabular}

$\mathrm{Na}$ transformação de referencial das velocidades do ITRF2008 para ITRF2005, apenas a velocidade $\left(\mathrm{V}_{\mathrm{X}}\right)$ é alterada, pois com exceção da variação temporal da translação em torno do eixo $\mathrm{X}$, todas as demais são iguais a zero.

Tabela 2: Parâmetros de transformação do ITRF2005 para ITRF2000 - época 2000,0.

\begin{tabular}{|c|c|c|c|c|c|c|c|}
\hline & $\mathrm{T}_{\mathrm{X}}(\mathbf{m})$ & $\mathrm{T}_{\mathrm{Y}}(\mathrm{m})$ & $\mathbf{T}_{\mathrm{Z}}(\mathbf{m})$ & $\mathbf{D}(\mathbf{p p b})$ & $\mathbf{R}_{\mathrm{X}}$ (mas) & $\mathbf{R}_{\mathbf{Y}}$ (mas) & $\mathbf{R}_{\mathrm{Z}}$ (mas) \\
\hline $\begin{array}{c}\text { Parâmetros e } \\
\text { precisões }\end{array}$ & 0,0001 & $-0,0008$ & $-0,0058$ & 0,40 & 0,000 & 0,000 & 0,000 \\
\hline \pm & 0,0003 & 0,0003 & 0,0003 & 0,05 & 0,012 & 0,012 & 0,012 \\
\hline \multirow{2}{*}{$\begin{array}{c}\text { Variações } \\
\text { temporais e } \\
\text { precisões }\end{array}$} & $\dot{\mathrm{T}}_{\mathrm{x}}$ (m/ano) & $\dot{\mathrm{T}}_{\mathrm{Y}}(\mathrm{m} / \mathrm{ano})$ & $\dot{\mathrm{T}}_{\mathrm{Z}}$ (m/ano) & $\dot{\mathbf{D}}(\mathbf{p p b} / \mathbf{a n o}$ & $\dot{\mathbf{R}}_{\mathbf{X}}(\mathbf{m a s} /$ ano $)$ & $\dot{\mathbf{R}}_{\mathbf{Y}}($ mas/ano $)$ & $\dot{\mathbf{R}}_{\mathrm{Z}}($ mas/ano $)$ \\
\hline & $-0,0002$ & 0,0001 & $-0,0018$ & 0,08 & 0,000 & 0,000 & 0,000 \\
\hline \pm & 0,0003 & 0,0003 & 0,0003 & 0,05 & 0,012 & 0,012 & 0,012 \\
\hline
\end{tabular}

Fonte: http://itrf.ensg.ign.fr/ITRF_solutions/2005/tp_05-00.php

A atualização das coordenadas das estações da época $\left(\mathrm{t}_{0}\right)$ para a época de interesse $(\mathrm{t})$ pode ser realizada de acordo com a Equação 4 (Petit and Luzum 2010); (SIRGAS 2014):

$$
\left[\begin{array}{l}
\mathrm{X}(\mathrm{t}) \\
\mathrm{Y}(\mathrm{t}) \\
\mathrm{Z}(\mathrm{t})
\end{array}\right]=\left[\begin{array}{c}
\mathrm{X}\left(\mathrm{t}_{0}\right) \\
\mathrm{Y}\left(\mathrm{t}_{0}\right) \\
\mathrm{Z}\left(\mathrm{t}_{0}\right)
\end{array}\right]+\left[\begin{array}{c}
\mathrm{V}_{\mathrm{X}} \\
\mathrm{V}_{\mathrm{Y}} \\
\mathrm{V}_{\mathrm{Z}}
\end{array}\right] \cdot \Delta \mathrm{t}
$$

Em que:

$\Delta \mathrm{t}$ : é a diferença entre as épocas destino $(\mathrm{t})$ e origem $\left(\mathrm{t}_{0}\right)$ das coordenadas cartesianas geocêntricas;

t: época de interesse para a determinação das coordenadas; 
$\mathrm{t}_{0}$ : época de origem das coordenadas cartesianas geocêntricas;

$\mathrm{X}(\mathrm{t}), \mathrm{Y}(\mathrm{t}), \mathrm{Z}(\mathrm{t})$; coordenadas cartesianas geocêntricas de uma estação na época de destino;

$\mathrm{X}\left(\mathrm{t}_{0}\right), \mathrm{Y}\left(\mathrm{t}_{0}\right), \mathrm{Z}\left(\mathrm{t}_{0}\right)$ : coordenadas cartesianas geocêntricas de uma estação na época de origem; $\mathrm{e}$

$\mathrm{V}_{\mathrm{X}}, \mathrm{V}_{\mathrm{Y}}, \mathrm{V}_{\mathrm{Z}}$ : velocidades cartesianas da estação no mesmo referencial das coordenadas na época $\left(\mathrm{t}_{0}\right)$.

\section{Propagação de variância}

As precisões das coordenadas e velocidades após realização da mudança de referencial e atualização foram estimadas com uso da propagação de variância conforme descrito nas seções de 3.1 a 3.4 .

\subsection{Propagação de variância na atualização de coordenadas}

As precisões das coordenadas atualizadas podem ser obtidas com o emprego da propagação de variância nas equações (5), (6) e (7). Para isso, consideram-se as precisões das coordenadas na época de origem $\left(\mathrm{t}_{0}\right)$ e das velocidades.

As precisões das coordenadas atualizadas para uma época de interesse podem ser obtidas com base na Equação 5.

$$
\sum_{\mathbf{x}(\mathrm{t})}=\mathbf{J} \sum_{\mathbf{x}\left(\mathrm{t}_{\mathrm{o}}\right), \mathbf{v}} \mathbf{J}^{\mathrm{T}}
$$

Sendo:

$$
\begin{aligned}
\mathbf{J} & =\left[\begin{array}{cccccc}
1 & 0 & 0 & \left(\mathrm{t}-\mathrm{t}_{0}\right) & 0 & 0 \\
0 & 1 & 0 & 0 & \left(\mathrm{t}-\mathrm{t}_{0}\right) & 0 \\
0 & 0 & 1 & 0 & 0 & \left(\mathrm{t}-\mathrm{t}_{0}\right)
\end{array}\right]_{3 \times 6} \\
\sum_{\mathbf{x}\left(\mathrm{t}_{0}\right), v} & =\operatorname{diag}\left[\begin{array}{llllll}
\sigma_{\mathrm{X}\left(\mathrm{t}_{0}\right)}^{2} & \sigma_{\mathrm{Y}\left(\mathrm{t}_{0}\right)}^{2} & \sigma_{Z\left(\mathrm{t}_{0}\right)}^{2} & \sigma_{\mathrm{V}_{\mathrm{X}}}^{2} & \sigma_{\mathrm{V}_{\mathrm{Y}}}^{2} & \sigma_{\mathrm{V}_{\mathrm{Z}}}^{2}
\end{array}\right]_{6}
\end{aligned}
$$

Em que:

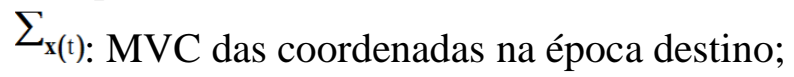

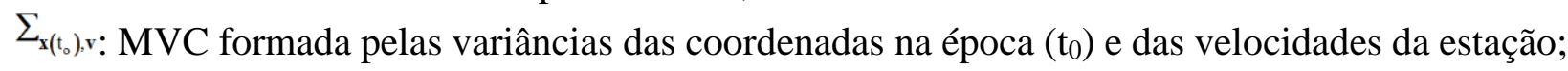

$\mathrm{e}$

J: Matriz formada pelas derivadas parciais de primeira ordem das equações em relação às coordenadas na época $\left(t_{0}\right)$ e das velocidades. 


\subsection{Propagação de variância na atualização de parâmetros de transformação}

Os parâmetros de transformação são determinados em uma época específica $\left(t_{k}\right)$ e caso seja necessário atualizá-los para uma época $\left(\mathrm{t}_{0}\right)$, torna-se necessário realizar propagação de variância para estimar as precisões dos parâmetros atualizados.

As precisões parâmetros de transformação atualizados podem ser obtidas com o modelo de propagação de variância dado por (8):

$$
\sum_{p(t)}=\mathbf{J} \sum_{p\left(t_{o}\right) \dot{p}} \mathbf{J}^{\mathrm{T}}
$$

Em que:

$\Sigma_{\mathrm{p}(\mathrm{t})}:$ MVC dos parâmetros de transformações atualizados;

$\Sigma_{\mathrm{p}\left(\mathrm{t}_{\mathrm{o}}\right) \mathrm{p}}$ : MVC dos parâmetros de transformações na época $\left(\mathrm{t}_{0}\right)$ e suas variações temporais; e

J : Matriz formada pelas derivadas das equações em relação aos parâmetros e suas variações temporais.

A matriz (J) da Equação 8 é obtida derivando a Equação 2 em relação aos parâmetros e suas variações temporais e é dada por (9).

$$
\mathbf{J}=\left[\begin{array}{cccccccccccccc}
1 & 0 & 0 & 0 & 0 & 0 & 0 & \left(\mathrm{t}_{0}-\mathrm{t}_{\mathrm{k}}\right) & 0 & 0 & 0 & 0 & 0 & 0 \\
0 & 1 & 0 & 0 & 0 & 0 & 0 & 0 & \left(\mathrm{t}_{0}-\mathrm{t}_{\mathrm{k}}\right) & 0 & 0 & 0 & 0 & 0 \\
0 & 0 & 1 & 0 & 0 & 0 & 0 & 0 & 0 & \left(\mathrm{t}_{0}-\mathrm{t}_{\mathrm{k}}\right) & 0 & 0 & 0 & 0 \\
0 & 0 & 0 & 1 & 0 & 0 & 0 & 0 & 0 & 0 & \left(\mathrm{t}_{0}-\mathrm{t}_{\mathrm{k}}\right) & 0 & 0 & 0 \\
0 & 0 & 0 & 0 & 1 & 0 & 0 & 0 & 0 & 0 & 0 & \left(\mathrm{t}_{0}-\mathrm{t}_{\mathrm{k}}\right) & 0 & 0 \\
0 & 0 & 0 & 0 & 0 & 1 & 0 & 0 & 0 & 0 & 0 & 0 & \left(\mathrm{t}_{0}-\mathrm{t}_{\mathrm{k}}\right) & 0 \\
0 & 0 & 0 & 0 & 0 & 0 & 1 & 0 & 0 & 0 & 0 & 0 & 0 & \left(\mathrm{t}_{0}-\mathrm{t}_{\mathrm{k}}\right)
\end{array}\right]_{7 \times \mathrm{x} 14}
$$

A MVC $\left({ }^{\left.\sum_{p\left(t_{0}\right) \mathrm{p}}\right)}\right)$ na época $\left(t_{0}\right)$ e de suas variações temporais pode ser dada por:

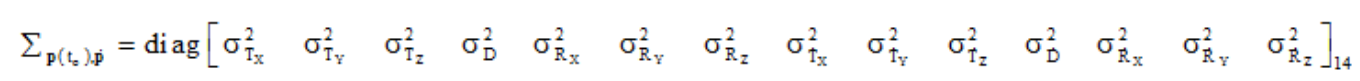

Em (10) as covariâncias entre os parâmetros e variações temporais foram consideradas como sendo iguais a zero.

\subsection{Propagação de variância na transformação de sistemas de referência das coordenadas}

As precisões das coordenadas no sistema de referência destino podem ser obtidas pela aplicação da propagação de variância na equação 1 resultando em:

Em que:

$$
\Sigma_{\mathrm{xd}}=\mathbf{J} \sum_{\mathrm{xo}, \mathrm{p}} \mathbf{J}^{\mathrm{T}}
$$

$\Sigma_{x d}:$ MVC das coordenadas no sistema de referência destino;

Bol. Ciênc. Geod., sec. Artigos, Curitiba, v. 21, nº 3, p.590 - 609, jul-set, 2015. 
$\Sigma_{\mathrm{xo}, \mathrm{p}}$ : MVC dos parâmetros de transformação e das coordenadas no sistema origem; e

J: Matriz formada pelas derivadas parciais da Equação 1 em relação aos sete parâmetros de transformação e às coordenadas no referencial origem.

A matriz ( J) é obtida a partir da derivada parcial da Equação 1 em relação às variáveis (coordenadas e parâmetros de transformação na época $\left({ }^{\mathrm{t}_{0}}\right)$ ) e tem a forma apresentada em (12).

$$
\mathbf{J}=\left[\begin{array}{cccccccccc}
1+\mathrm{D} & -\mathrm{Rz} & \mathrm{Ry} & 1 & 0 & 0 & \mathrm{X}_{0} & 0 & \mathrm{Z}_{0} & -\mathrm{Y}_{0} \\
\mathrm{Rz} & 1+\mathrm{D} & -\mathrm{Rx} & 0 & 1 & 0 & \mathrm{Y}_{0} & -\mathrm{Z}_{0} & 0 & \mathrm{X}_{0} \\
-\mathrm{Ry} & \mathrm{Rx} & 1+\mathrm{D} & 0 & 0 & 1 & \mathrm{Z}_{0} & \mathrm{Y}_{0} & -\mathrm{X}_{0} & 0
\end{array}\right]_{3 \times 10}
$$

A MVC $\left(\Sigma_{\mathrm{xop}, \mathrm{p}}\right)$ pode ser diagonal, caso as covariâncias entre os parâmetros e entre as coordenadas na rede origem, época $t_{0}$, sejam nulas.

Admitindo a não existência de covariância entre os parâmetros e entre as coordenadas para uma estação, a MVC tomaria a forma (13):

$$
\Sigma_{\mathbf{x} 0, \mathrm{p}}=\operatorname{diag}\left[\begin{array}{llllllllll}
\sigma_{\mathrm{X}_{0}}^{2} & \sigma_{\mathrm{Y}_{0}}^{2} & \sigma_{Z_{0}}^{2} & \sigma_{\mathrm{T}_{\mathrm{X}}}^{2} & \sigma_{\mathrm{T}_{\mathrm{y}}}^{2} & \sigma_{\mathrm{T}_{z}}^{2} & \sigma_{\mathrm{D}}^{2} & \sigma_{\mathrm{R}_{\mathrm{X}}}^{2} & \sigma_{\mathrm{R}_{\mathrm{Y}}}^{2} & \sigma_{\mathrm{R}_{z}}^{2}
\end{array}\right]_{10}
$$

As precisões das coordenadas e das translações deverão estar em metros, o fator diferencial de escala é adimensional e as rotações diferenciais em radianos.

Se a época destino for diferente da época de referência dos parâmetros de transformação, deverá ser realizado inicialmente a propagação de variância de acordo com a Equação 8 para que as precisões dos parâmetros atualizados sejam empregadas em (11).

É importante realizar a propagação de variância na atualização das coordenadas e para que isso seja possível, é necessário o conhecimento das precisões das coordenadas e das velocidades empregadas para atualizá-las.

\subsection{Propagação de variância na transformação de referencial das velocidades}

As precisões das velocidades no sistema de referência destino foram estimadas a partir da propagação de variância na transformação entre sistemas de referência das velocidades, Equação 3 , tem-se que:

Em que:

$$
\sum_{\mathbf{v}_{\mathrm{d}}}=\mathbf{J} \sum_{\mathrm{v}_{\mathrm{o}}, \dot{\mathrm{p}}, \mathrm{x}_{\mathrm{o}}} \mathbf{J}^{\mathrm{T}}
$$

$\Sigma_{\mathrm{v}_{\mathrm{d}}}:$ MVC das velocidades no sistema de referência destino;

$\sum_{\mathrm{v}_{0}, \dot{\mathrm{p}}, \mathrm{x}_{0}}$ : MVC formada pelas variâncias das velocidades no sistema de referência origem, das variações temporais dos parâmetros de transformação e das coordenadas da estação no sistema de referência origem; e

J: Matriz das derivadas parciais das equações em relação às variáveis que possuem variância conhecidas. 
A matriz $(\mathbf{J})$ tem a forma apresentada em (15).

$$
\mathbf{J}=\left[\begin{array}{ccccccccccccc}
1 & 0 & 0 & 1 & 0 & 0 & \mathrm{X}_{0} & 0 & \mathrm{Z}_{0} & -\mathrm{Y}_{0} & \dot{\mathrm{D}} & -\dot{\mathrm{R}}_{\mathrm{Z}} & \dot{\mathrm{R}}_{\mathrm{Y}} \\
0 & 1 & 0 & 0 & 1 & 0 & \mathrm{Y}_{0} & -\mathrm{Z}_{0} & 0 & \mathrm{X}_{0} & \dot{\mathrm{R}}_{\mathrm{Z}} & \dot{\mathrm{D}} & -\dot{\mathrm{R}}_{\mathrm{X}} \\
0 & 0 & 1 & 0 & 0 & 1 & \mathrm{Z}_{0} & \mathrm{Y}_{0} & -\mathrm{X}_{0} & 0 & -\dot{\mathrm{R}}_{\mathrm{Y}} & \dot{\mathrm{R}}_{\mathrm{X}} & \dot{\mathrm{D}}
\end{array}\right]_{3 \times 13}
$$

$\operatorname{A~MVC~}\left(\sum_{\mathrm{V}_{0}, \hat{p}, \mathbf{x}_{\circ}}\right)$ é diagonal, pois as covariâncias entre as velocidades, as variações temporais dos parâmetros de transformação e as coordenadas no sistema de referência de origem são iguais a zero e tem a forma (16):

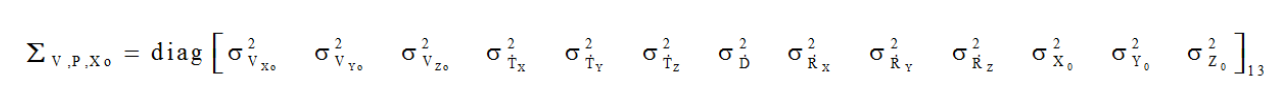

As precisões das variações temporais das translações deverão estar em metros por ano, o fator diferencial de escala adimensional, as variações das rotações diferenciais em radianos por ano e as coordenadas em metros.

\section{Estações e fluxograma da metodologia adotada}

As 11 estações utilizadas neste trabalho estão distribuídas em três placas tectônicas (NorteAmericana, Sul-Americana e Eurásia) e foram escolhidas de forma que tivessem suas coordenadas nas realizações ITRF2008 (2005,0), ITRF2005 (2000,0) e ITRF2000 (1997,0). Na Tabela 3 encontram-se as estações e respectivas placas tectônicas as quais pertencem.

Tabela 3: Estações e respectivas localizações.

\begin{tabular}{ccc}
\hline Localidade & Identificador & Placa tectônica \\
\hline Fairbanks & FAIR & Norte-Americana \\
\hline Yellowknife & YELL & Norte-Americana \\
\hline Goldstone & GOLD & Norte-Americana \\
\hline Kourou & KOUR & Sul-Americana \\
\hline Fortaleza & FORT & Sul-Americana \\
\hline Brasília & BRAZ & Sul-Americana \\
\hline Santiago & SANT & Sul-Americana \\
\hline Rio Grande & RIOG & Sul-Americana \\
\hline Kootwijk & KOSG & Eurásia \\
\hline Brussels & BRUS & Eurásia \\
\hline Matera & MATE & Eurásia \\
\hline
\end{tabular}

$\mathrm{Na}$ Figura 1 pode-se visualizar as posições aproximadas das 11 estações e as divisas das placas tectônicas. 


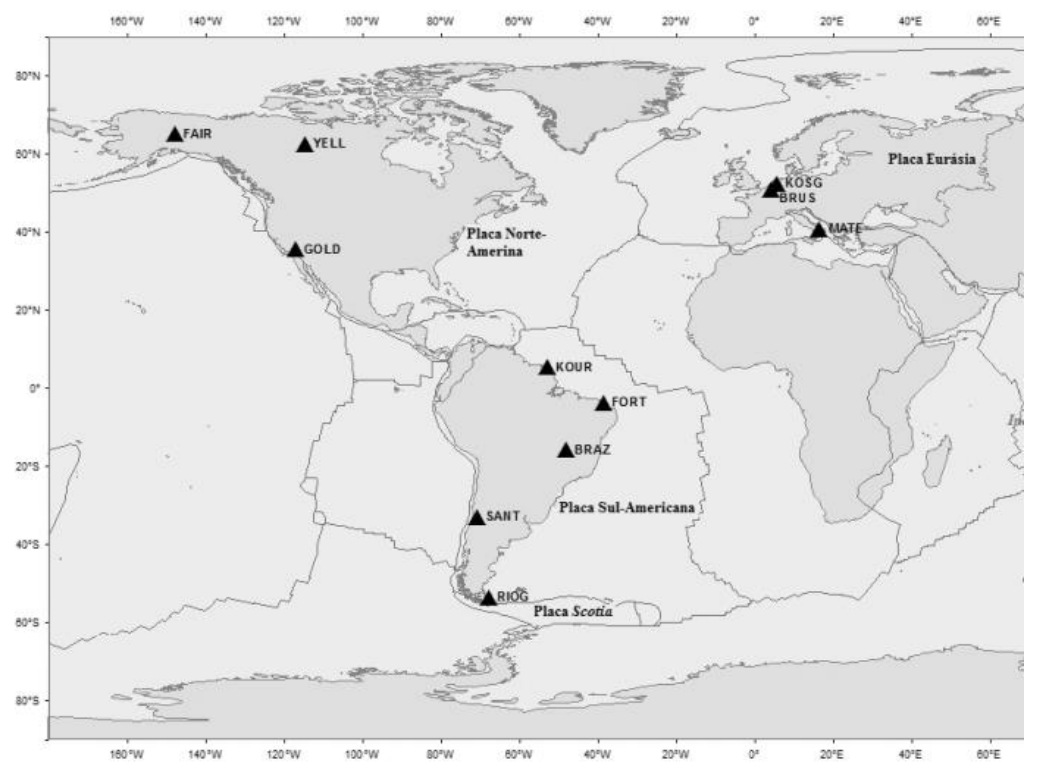

Figura 1: Distribuição espacial das estações.

A Figura 2 ilustra o procedimento adotado para obtenção das coordenadas e velocidades nos referenciais e épocas de interesse.

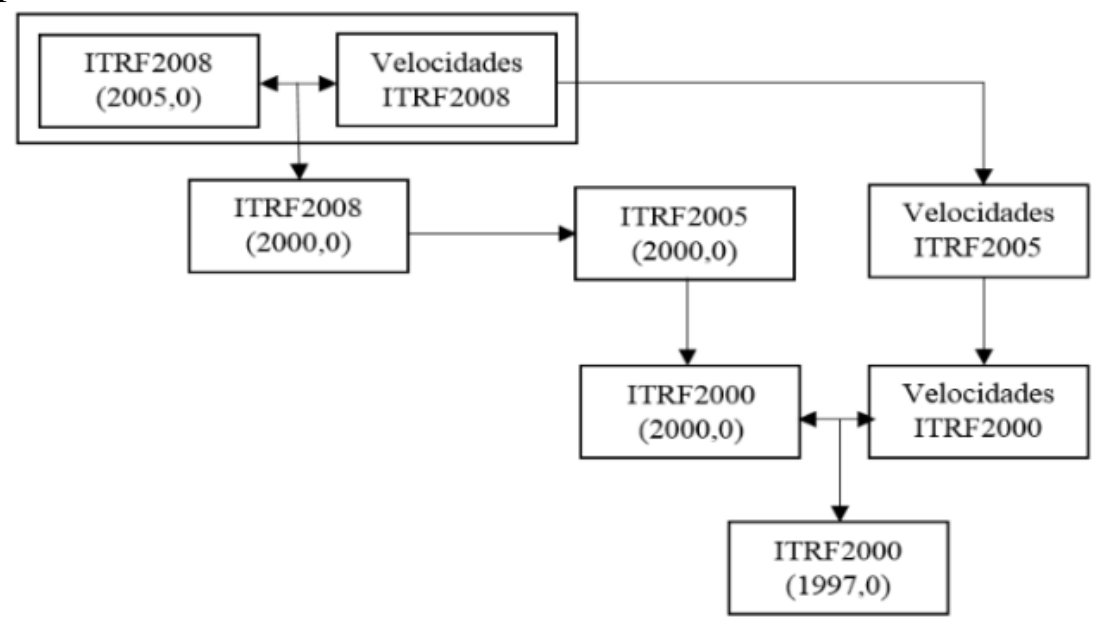

Figura 2: Etapas seguidas para determinação das coordenadas e velocidades.

Conforme ilustra a Figura 2, a partir das coordenadas e velocidades no ITRF2008, época 2005,0 determinou-se as coordenadas das estações no ITRF2008, época 2000,0 com base na Equação 4.

As coordenadas no ITRF2005, época 2000,0 foram determinadas realizando inicialmente a atualização dos parâmetros de transformação do ITRF2008 para ITRF2005 da época 2005,0 para a época 2000,0 com o uso da Equação 2. Na sequência, empregou-se a Equação 1 para obtenção das coordenadas no ITRF2005, época 2000,0.

A determinação das coordenadas no ITRF2000, época 2000 foi realizada com base na utilização a Equação 1 e os parâmetros de transformação do ITRF2005 para o ITRF2000, época 2000,0.

Para atualizar as coordenadas no ITRF2000, da época 2000,0 para a época 1997,0 foi necessário realizar a mudança de referencial das velocidades do ITRF2008 para ITRF2005 e deste para o ITRF2000 com o emprego da Equação 3. Com as coordenadas e velocidades no mesmo referencial pôde-se atualizar as coordenadas para a época de interesse. 
As discrepâncias em termos de coordenadas geodésicas (latitude, longitude e altitude elipsoidal) no ITRF2005, época 2000,0 e no ITRF2000, época 1997,0 foram calculadas com base na comparação das coordenadas obtidas com as transformações e atualizações com as divulgadas nas soluções oficiais.

As precisões das coordenadas e velocidades no ITRF2005 e no ITRF2000, nas épocas 2000,0 e 1997,0, respectivamente, foram obtidas com o emprego de propagação de variância na atualização de parâmetros, das coordenadas e nas transformações de referenciais das coordenadas e velocidades.

\section{Experimentos e análise dos resultados}

Todos os algoritmos para atualização das coordenadas e mudança de referencial bem como a estimativa das precisões foram desenvolvidos em ambiente Matlab. Para exemplificar os procedimentos descritos nas seções anteriores apresentam-se os resultados para a estação BRAZ.

O Mesmo procedimento foi aplicado para as 10 estações restantes.

\subsection{Determinação das coordenadas, velocidades e respectivas precisões para a estação BRAZ nos ITRF2005, época 2000 e ITRF2000, época 1997,0 a partir do ITRF2008, época 2005.}

As coordenadas, velocidades e precisões da estação BRAZ nas realizações ITRF2008, ITRF2005 e ITRF2000, nas épocas 2005,0, 2000,0 e 1997,0, estão apresentadas na Tabela 4 e foram obtidas no site $<<$ http://itrf.ensg.ign.fr/ITRF_solutions/index.php $>>$.

Tabela 4: Coordenadas, velocidades e precisões nas realizações ITRF2000, ITRF2005 e ITRF2008.

\begin{tabular}{ccccccc}
\hline Solução & $\mathbf{X} / \mathbf{V x}$ & $\mathbf{Y} / \mathbf{V y}$ & $\mathbf{Z} / \mathbf{V z}$ & Sigma & Sigma & Sigma \\
\cline { 2 - 7 } Época & $\mathbf{m} / \mathbf{m} / \mathbf{a n o}$ & $\mathbf{m} / \mathbf{m} / \mathbf{a n o}$ & $\mathbf{m} / \mathbf{m} / \mathbf{a n o}$ & $\mathbf{m}$ & $\mathbf{m}$ & $\mathbf{m}$ \\
\hline ITRF2000 & 4115014,087 & $-4550641,532$ & $-1741444,061$ & 0,003 & 0,003 & 0,002 \\
$(1997,0)$ & 0,0005 & $-0,0063$ & 0,0115 & 0,0016 & 0,0017 & 0,0008 \\
\hline ITRF2005 & 4115014,083 & $-4550641,541$ & $-1741444,022$ & 0,001 & 0,001 & 0,001 \\
$(2000,0)$ & 0,0002 & $-0,0046$ & 0,0124 & 0,0002 & 0,0002 & 0,0001 \\
\hline ITRF2008 & 4115014,074 & $-4550641,559$ & $-1741443,951$ & 0,001 & 0,001 & 0,001 \\
$(2005,0)$ & $-0,0006$ & $-0,0049$ & 0,0121 & 0,0001 & 0,0001 & 0,0000 \\
\hline
\end{tabular}

As coordenadas e precisões da estação BRAZ no ITRF2008, época 2000,0 foram obtidas com base na equação (4) sendo iguais a:

$\mathrm{X}_{\text {ITRF } 2008}(2000,0)==4115014,077 \mathrm{~m} ; \mathrm{Y}_{\text {ITRF } 2008}(2000,0)==-4550641,5345 \mathrm{~m} ; \mathrm{Z}_{\text {ITRF 2008 }}(2000,0)==-$
$1741444,0115 \mathrm{~m} . \sigma_{\mathrm{X}_{\text {ITRF } 2008}(2000,0)}=0,0011 \mathrm{~m} ; \sigma_{\mathrm{Y}_{\text {ITRF 2008 }}(2000,0)}=0,001 \mathrm{~m}_{\mathrm{m}} \sigma_{\mathrm{Z}_{\text {ITRF 2008 }}(2000,0)}=0,0010 \mathrm{~m}$. 
As coordenadas no ITRF2005, época 2000,0 foram determinadas com base na equação (1) e nos parâmetros de transformação disponíveis na Tabela 1 atualizados para a época 2000,0 com o uso da equação (2). Para atualizar os parâmetros deve-se atentar para as unidades empregadas. $\mathrm{O}$ fator de escala ser dado em partes por bilhão, deve ser dividido por 1 bilhão e as rotações diferenciais em milissegundo de arco deverão ser convertidas para radianos, da seguinte forma:

$$
\begin{aligned}
& 1 \mathrm{ppb}=1 \times \frac{1}{1.000 .000 .000} \text { partes }=1 \times 10^{-9} 1 \mathrm{ppb}=1 \times \frac{1}{1.000 .000 .000} \text { partes }=1 \times 10^{-9} . \\
& 1 \mathrm{mas}=1 \times \frac{1}{1000} \times \frac{\pi}{60 \times 60 \times 180} \text { radianos }=4,8481368111 \times 10^{-9} \\
& 1 \mathrm{mas}=1 \times \frac{1}{1000} \times \frac{\pi}{60 \times 60 \times 180} \text { radianos }=4,8481368111 \times 10^{-9} .
\end{aligned}
$$

Os parâmetros de transformação do ITRF2008 para ITRF2005 atualizados para a época 2000,0 e respectivas precisões são iguais a:

$\mathrm{T}_{\mathrm{X}}=\mathrm{T}_{\mathrm{X}}=-0,002 \mathrm{~m} ; \mathrm{T}_{\mathrm{Y}}=\mathrm{T}_{\mathrm{Y}}=-0,0009 \mathrm{~m} ; \mathrm{T}_{\mathrm{Z}}=\mathrm{T}_{\mathrm{Z}}=-0,0047 \mathrm{~m} ; \mathrm{D}=\mathrm{D}=0,94 \times 10^{-9}$ $0,94 \times 10^{-9} ; \mathrm{R}_{\mathrm{X}} \mathrm{R}_{\mathrm{X}}=0 ; \mathrm{R}_{\mathrm{Y}} \mathrm{R}_{\mathrm{Y}}=0 ; \mathrm{R}_{\mathrm{Z}} \mathrm{R}_{\mathrm{Z}}=0$.

$\sigma \mathrm{T}_{\mathrm{X}}=\sigma \mathrm{T}_{\mathrm{X}}=0,0010 \mathrm{~m} ; \sigma \mathrm{T}_{\mathrm{Y}}=\sigma \mathrm{T}_{\mathrm{Y}}=0,0010 \mathrm{~m} ; \sigma \mathrm{T}_{\mathrm{Z}}=\sigma \mathrm{T}_{\mathrm{Z}}=0,0010 \mathrm{~m} ; \sigma \mathrm{D}=0,1529$ $\sigma \mathrm{D}=0,1529 \mathrm{ppb} ; \sigma \mathrm{R}_{\mathrm{X}} \sigma \mathrm{R}_{\mathrm{X}}=0,0408$ mas/ano; $\sigma \mathrm{R}_{\mathrm{Y}} \sigma \mathrm{R}_{\mathrm{Y}}=0,0408$ mas/ano; $\mathrm{e}^{\sigma \mathrm{R}_{\mathrm{Z}} \sigma \mathrm{R}_{\mathrm{Z}}=0,0408}$ mas/ano.

As coordenadas da estação BRAZ no ITRF2005, época 2000 foram obtidas empregando a equação (1) e os parâmetros de transformação do ITRF2008 para o ITRF2005 atualizados para a época 2000,0 e são iguais a:

$$
\begin{aligned}
& X_{\text {ITRF2005 }}(2000,0)=4115014,0789 \mathrm{~m} ; Y_{\text {ITRF 2005 }}(2000,0)=-4550641,5397 \mathrm{~m} ; \\
& Z_{\text {ITRF 2005 }}(2000,0)=-1741444,01784 \mathrm{~m} . \quad \sigma_{\mathrm{X}_{\text {TRF 2005 }(2000,0)}=0,0019} \mathrm{~m} ; \quad \sigma_{\mathrm{I}_{\text {ITRF 2005 }}(2000,0)}=0,0019 \mathrm{~m} ; \\
& \sigma_{Z_{\text {ITRF 2005 }}(2000,0)}=0,0019 \mathrm{~m} .
\end{aligned}
$$

A coordenadas no ITRF2000, época 2000,0 foram determinadas com base na Equação 1 e nos parâmetros de transformação disponíveis na Tabela 2 e são iguais a:

$$
\begin{aligned}
& X_{\text {ITRF 2000 }}(2000,0)=4115014,0806 \mathrm{~m} ; Y_{\text {ITRF 2000 }}(2000,0)=-4550641,5423 \mathrm{~m} ; \\
& Z_{\text {ITRF 2000 }}(2000,0)=-1741444,0243 \mathrm{~m} . \quad \sigma_{X_{\text {ITRF 2000 }(2000,0)}=0,0020} \mathrm{~m} \sigma_{\mathrm{Y}_{\text {ITRF 2000 }}(2000,0)}=0,0019 \quad \mathrm{~m} ; \\
& \sigma_{Z_{\text {ITRF 2000 }}(2000,0)}=0,0020 \mathrm{~m} .
\end{aligned}
$$

As coordenadas no ITRF2000, época 1997,0 foram obtidas com base na Equação 4. Porém, antes de utilizá-las, foi necessário realizar a transformação do referencial das velocidades do ITRF2008 para ITRF2005 e deste para o ITRF2000 para compatibilizar os referenciais das coordenadas e velocidades. As velocidades da estação BRAZ nos referenciais ITRF2005 e ITRF2000 foram calculadas com o emprego da Equação 3. As variações temporais dos parâmetros de transformação estão disponíveis nas Tabelas 2 e 3 .

As velocidades e precisões da estação BRAZ no ITRF2005 são iguais a:

$V_{X} V_{X}=-0,0003 \mathrm{~m} /$ ano; $V_{Y} V_{Y}=-0,0049 \mathrm{~m} /$ ano; $V_{Z} V_{Z}=0,0121 \mathrm{~m} /$ ano. 
$\sigma_{\mathrm{Vx}}=0,0003 \mathrm{~m} / \mathrm{ano} ; \sigma_{\mathrm{vy}}=0,0003 \mathrm{~m} / \mathrm{ano} ; \sigma_{\mathrm{Vz}}=0,0003 \mathrm{~m} / \mathrm{ano}$.

As velocidades e precisões da estação BRAZ nos ITRF2000 são iguais a:

$\mathrm{V}_{\mathrm{X}} \mathrm{V}_{\mathrm{X}}=-0,0002 \mathrm{~m} / \mathrm{ano} ; \mathrm{V}_{\mathrm{Y}} \mathrm{V}_{\mathrm{Y}}=-0,0052 \mathrm{~m} / \mathrm{ano} ; \mathrm{V}_{\mathrm{Z}} \mathrm{V}_{\mathrm{Z}}=0,0102 \mathrm{~m} / \mathrm{ano}$.

$\sigma_{\mathrm{Vx}}=0,0005 \mathrm{~m} / \mathrm{ano} ; \sigma_{\mathrm{Vy}}=0,0005 \mathrm{~m} / \mathrm{ano} ; \sigma_{\mathrm{Vz}}=0,0005 \mathrm{~m} / \mathrm{ano}$.

A coordenadas e precisões no ITRF2000, época 1997,0 são iguais a:

$\mathrm{X}_{\text {ITRF 2000 }}(1997,0)=4115014,0806 \mathrm{~m} ; \mathrm{Y}_{\text {ITRF2000 }}(1997,0)=-4550641,5423 \mathrm{~m}$;

$\mathrm{Z}_{\text {ITRF2000 }}(1997,0)=-1741444,0243 \mathrm{~m} . \sigma_{\mathrm{X}_{\text {ITRF 2000 }}(1997,0)}=0,0025 \mathrm{~m} ; \sigma_{\mathrm{Y}_{\text {ITRF 2000 }}(1997,0)}=0,0025 \mathrm{~m}$;

$\sigma_{\mathrm{Z}_{\mathrm{ITRF} 2000}(1997,0)}=0,0026 \mathrm{~m}$.

\subsection{Análise dos resultados}

A Figura 3 ilustra as discrepâncias para as coordenadas geodésicas (latitude $\left(\prod\right)$, longitude $(\lfloor)$ e altitude elipsoidal (h)) e para as resultantes planimétricas e tridimensional das coordenadas no ITRF2008, época 2000,0 em relação às coordenadas de referência no mesmo referencial, mas na época 2005,0.

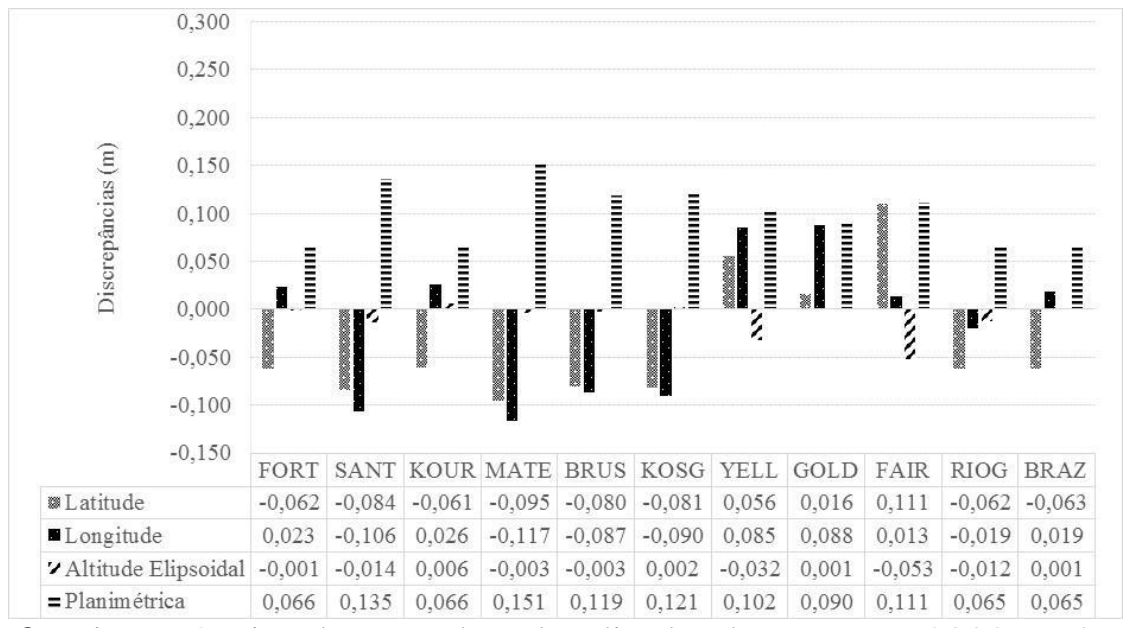

Figura 3: Discrepâncias das coordenadas divulgadas no ITRF2008 na época 2005,0 e atualizadas para a época 2000,0 .

A Tabela 5 apresenta as velocidades resultantes no ITRF2008 das estações empregadas. 
Tabela 5: Velocidade resultante.

\begin{tabular}{cc}
\hline Estação & $\mathbf{V}(\mathbf{m} / \mathbf{a n o})$ \\
\hline FORT & 0,013 \\
\hline SANT & 0,027 \\
\hline KOUR & 0,013 \\
\hline MATE & 0,030 \\
\hline BRUS & 0,024 \\
\hline KOSG & 0,024 \\
\hline YELL & 0,021 \\
\hline GOLD & 0,018 \\
\hline FAIR & 0,025 \\
\hline RIOG & 0,013 \\
\hline BRAZ & 0,013 \\
\hline
\end{tabular}

As discrepâncias foram obtidas considerando os deslocamentos das estações no período de 5 anos (2000 a 2005). Com base nas Figuras 1 e 3 pode-se verificar que as estações MATE e SANT estão localizadas nas proximidades de limite de placas e possuem maiores velocidades de descolamentos apresentaram deslocamentos planimétricos máximos de $0,151 \mathrm{~m}$ e $0,135 \mathrm{~m}$, respectivamente, e mínimos de 0,065 nas estações BRAZ e RIOG. Embora a estação RIOG esteja próxima a borda de placa, sua velocidade resultante é igual a BRAZ. A estação GOLD situada nas proximidades de divisa de placa apresenta velocidade resultante menor do que das estações MATE e SANT conforme pode ser visualizado na Tabela 5.

As maiores discrepâncias planimétricas foram detectadas nas estações localizadas na placa da Eurásia e da América do Norte. A discrepância altimétrica máxima, em termos absolutos, foi de 0,053 m na estação FAIR e mínima de 0,001 m para as estações FORT, GOLD e BRAZ

Com exceção da estação SANT presente na placa da América do Sul que apresentou deslocamento planimétrico decimétrico em 5 anos, as estações KOUR, FORT, BRAZ e RIOG tiveram movimento subdecimétrico.

Na Tabela 6 encontram-se os valores das estatísticas para as discrepâncias devido ao efeito da atualização das coordenadas no ITRF2008, época 2005 para a época 2000.

Tabela 6: Discrepâncias das coordenadas no ITRF2008, época 2005 em relação ao ITRF2008, época 2000,0.

\begin{tabular}{cccccc}
\hline Estatísticas & Latitude (m) & Longitude (m) & Altitude Elipsoidal (m) & Planimétrica (m) & Tridimensional (m) \\
\hline Máximo & 0,111 & 0,088 & 0,006 & 0,151 & 0,151 \\
\hline Mínimo & $-0,095$ & $-0,117$ & $-0,053$ & 0,065 & 0,065 \\
\hline Média & $-0,037$ & $-0,015$ & $-0,010$ & 0,099 & 0,101 \\
\hline Desvio Padrão & 0,067 & 0,074 & 0,018 & 0,031 & 0,032 \\
\hline EMQ & 0,074 & 0,073 & 0,020 & 0,104 & 0,105 \\
\hline
\end{tabular}

Os resultados mostram a importância em se considerar a variação espaço-temporal das estações, ou seja, ilustra a necessidade em se utilizar as velocidades das estações para atualizar suas coordenadas e desse modo considerar os movimentos das placas tectônicas.

Na Figura 4 pode-se observar as discrepâncias obtidas para as 11 estações calculadas a partir da comparação das coordenadas transformadas e atualizadas do ITRF2008, época 2005,0 para o ITRF2005, época 2000 com relação às obtidas no site do ITRF no mesmo referencial e época. 


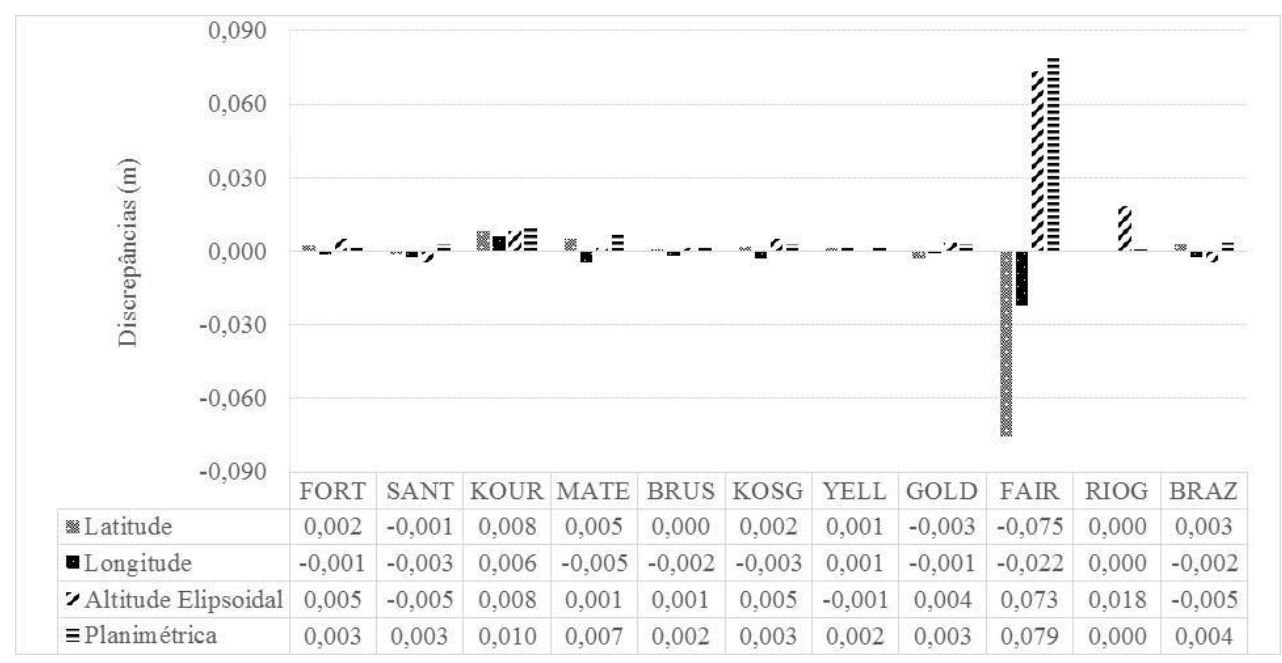

Figura 4: Discrepâncias das coordenadas calculadas no ITRF2005 e divulgada pelo IERS, ambas na época 2000,0.

Na Figura 4 pode-se observar com exceção das estações FAIR e KOUR que apresentaram discrepâncias planimétricas de $0,079 \mathrm{~m}$ e $0,010 \mathrm{~m}$, respectivamente, que nas demais foram encontradas discrepâncias planimétricas subcentimétricas.

O aumento na precisão na determinação posicional da estação FAIR na solução ITRF2008, época 2005 em relação a ITRF2005, época 2000,0 pode ser verificado nos dados iniciais. Talvez esse fato justifique as maiores discrepâncias planimétricas alcançadas para essa estação.

A maior discrepância foi encontrada na estação FAIR $(0,078 \mathrm{~m})$ e Pimenta et al. (2002) em experimentos haviam encontradas maiores discrepâncias posicionais em termos de coordenadas cartesianas geocêntricas no ITRF97 $(1997,0)$ para essa estação. Assim, esse fato sugere certa dificuldade na determinação posicional dessa estação.

$\mathrm{Na}$ Tabela 7 encontram-se um resumo das estatísticas para as discrepâncias provenientes da compatibilização de referencial e época para as 11 estações.

Tabela 7: Discrepâncias das coordenadas no ITRF2005, época 2000,0.

\begin{tabular}{cccccc}
\hline Estatísticas & Latitude (m) & Longitude (m) & Altitude Elipsoidal (m) & Planimétrica (m) & Tridimensional (m) \\
\hline Máximo & 0,008 & 0,006 & 0,073 & 0,078 & 0,108 \\
\hline Mínimo & $-0,075$ & $-0,022$ & $-0,005$ & 0,000 & 0,002 \\
\hline Média & $-0,005$ & $-0,003$ & 0,010 & 0,010 & 0,016 \\
\hline Desvio Padrão & 0,023 & 0,007 & 0,022 & 0,023 & 0,031 \\
\hline EMQ & 0,023 & 0,007 & 0,023 & 0,023 & 0,033 \\
\hline
\end{tabular}

Os valores apresentados na Tabela 7 estão afetados das maiores discrepâncias encontradas na estação FAIR, o que faz elevar os valores das estatísticas calculadas.

A Figura 5 ilustra as discrepâncias das estações no ITRF2000, época 1997,0 determinadas via mudança de referencial e atualização em relação às determinadas via solução IERS. 


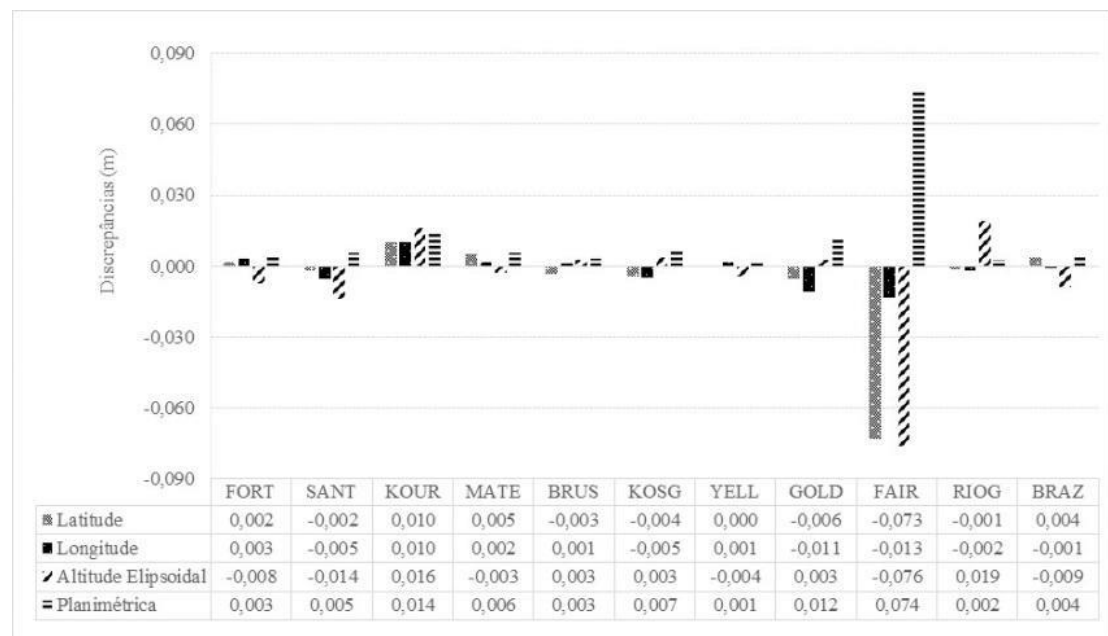

Figura 5: Discrepâncias das coordenadas calculadas no ITRF2000 e divulgada pelo IERS, ambas na época 1997,0.

Na Figura 5 pode-se observar que a discrepância planimétrica máxima encontrada foi na estação FAIR é de $0,074 \mathrm{~m}$ e com exceção das estações KOUR e GOLD que tiveram discrepâncias planimétricas de $0,014 \mathrm{~m}$ e $0,012 \mathrm{~m}$, respectivamente, as demais estações apresentaram valores no nível do milímetro. Foi verificado concordância milimétrica das coordenadas transformadas do ITRF2008, época 2005,0 para ITRF2000, época 1997,0 para estações localizadas no Brasil com as disponibilizadas pelo IERS. Das 11 estações, 72,73\% apresentaram discrepâncias planimétricas da ordem de $0,001 \mathrm{~m}$ a $0,007 \mathrm{~m}$ e $27,27 \%$ da ordem de $0,012 \mathrm{~m}$ a $0,074 \mathrm{~m}$.

Na Tabela 8 encontram-se um resumo das estatísticas para as discrepâncias provenientes da compatibilização de referencial e época para as 11 estações.

Tabela 8: Discrepâncias das coordenadas no ITRF2000, época 1997,0.

\begin{tabular}{cccccc}
\hline Estatísticas & Latitude (m) & Longitude (m) & Altitude Elipsoidal (m) & Planimétrica (m) & Tridimensional (m) \\
\hline Máximo & 0,010 & 0,010 & 0,019 & 0,074 & 0,107 \\
\hline Mínimo & $-0,073$ & $-0,013$ & $-0,076$ & 0,001 & 0,004 \\
\hline Média & $-0,006$ & $-0,002$ & $-0,006$ & 0,012 & 0,020 \\
\hline Desvio Padrão & 0,023 & 0,007 & 0,025 & 0,021 & 0,029 \\
\hline EMQ & 0,023 & 0,007 & 0,025 & 0,023 & 0,034 \\
\hline
\end{tabular}

A Figura 6 ilustra as discrepâncias calculadas a partir das coordenadas no ITRF2008, época 2005,0 em relação às coordenadas obtidas no ITRF2000, 1997 ambas disponibilizadas no site do IERS. 


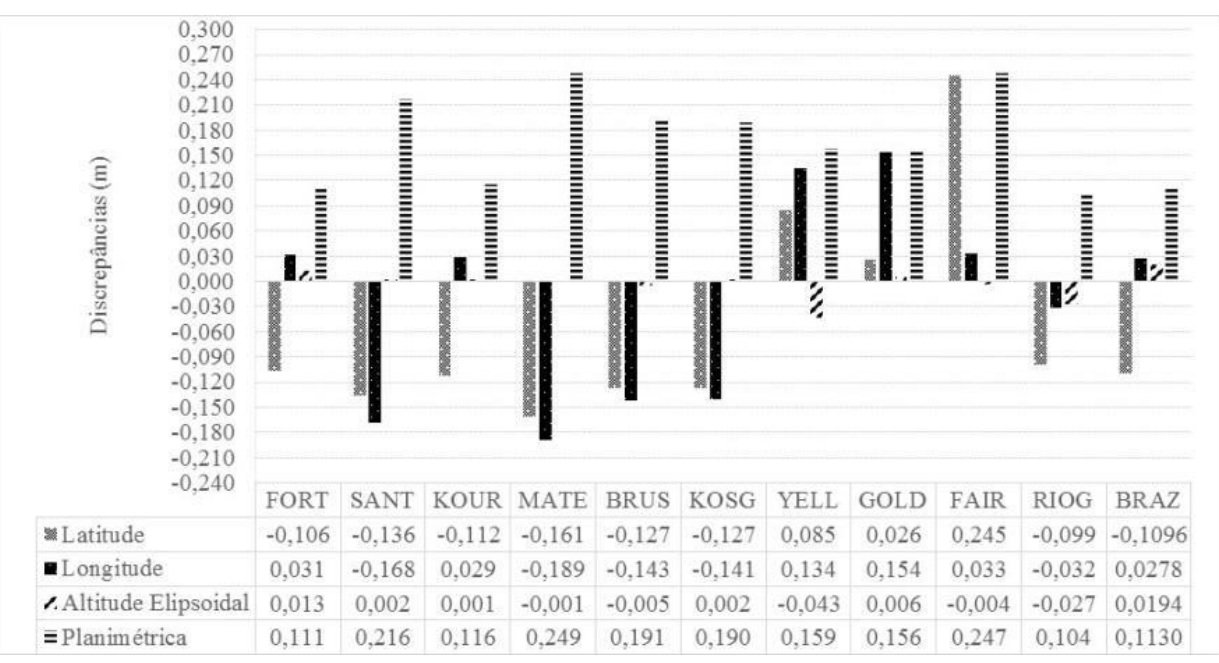

Figura 6:Discrepâncias das coordenadas divulgadas no ITRF2008 e ITRF2000, em relação, respectivamente, às épocas 2005,0 e 1997,0.

Comparando as informações presentes nas Figuras 3 e 6 verificam-se discrepâncias posicionais decimétricas se não forem contempladas mudança de referencial das coordenadas e velocidades bem como os deslocamentos das estações no tempo em consequência do movimento das placas tectônicas. Desse modo, pode-se obter alta precisão no posicionamento sem, contudo, obter acurácia.

As duas maiores discrepâncias planimétricas ocorreram nas estações MATE (0,249 m) e FAIR $(0,247 \mathrm{~m})$. As menores discrepâncias planimétricas ocorreram nas estações localizadas na placa da América do Sul.

Na Tabela 9 encontram-se um resumo das estatísticas para as discrepâncias provenientes da compatibilização de referencial e época para as 11 estações.

Tabela 9: Discrepâncias das coordenadas obtidas nas soluções ITRF2008, época 2005,0 e ITRF2000, época 1997,0.

\begin{tabular}{cccccc}
\hline Estatísticas & Latitude (m) & Longitude (m) & Altitude Elipsoidal (m) & Planimétrica (m) & Tridimensional (m) \\
\hline Máximo & 0,245 & 0,154 & 0,019 & 0,249 & 0,249 \\
\hline Mínimo & $-0,161$ & $-0,189$ & $-0,043$ & 0,104 & 0,107 \\
\hline Média & $-0,057$ & $-0,024$ & $-0,003$ & 0,168 & 0,169 \\
\hline Desvio Padrão & 0,125 & 0,120 & 0,018 & 0,054 & 0,054 \\
\hline EMQ & 0,132 & 0,117 & 0,017 & 0,176 & 0,177 \\
\hline
\end{tabular}

As discrepâncias encontradas na Tabela 9 ilustram os efeitos combinados dos deslocamentos das placas tectônicas, da diferença de referencial das coordenadas e da melhoria da qualidade da determinação posicional obtida em cada nova realização do ITRS pelo IERS.

Foi verificado com base nas coordenadas nos ITRF2008, época 2005 e ITRF2005, época 2000,0 obtidas pelo IERS que 54,55\% das discrepâncias planimétricas são centimétricas e 45,45\% decimétricas. Esse fato, coloca em evidência a necessidade e a importância da consideração da variação espaço-temporal das coordenadas das estações.

Nas Figuras 7 e 8 podem ser observados desvios-padrão das coordenadas calculados com o emprego da propagação de variância nos procedimentos de atualização e transformação entre sistemas de referência das coordenadas e velocidades e os provenientes da solução ITRF do IERS. 


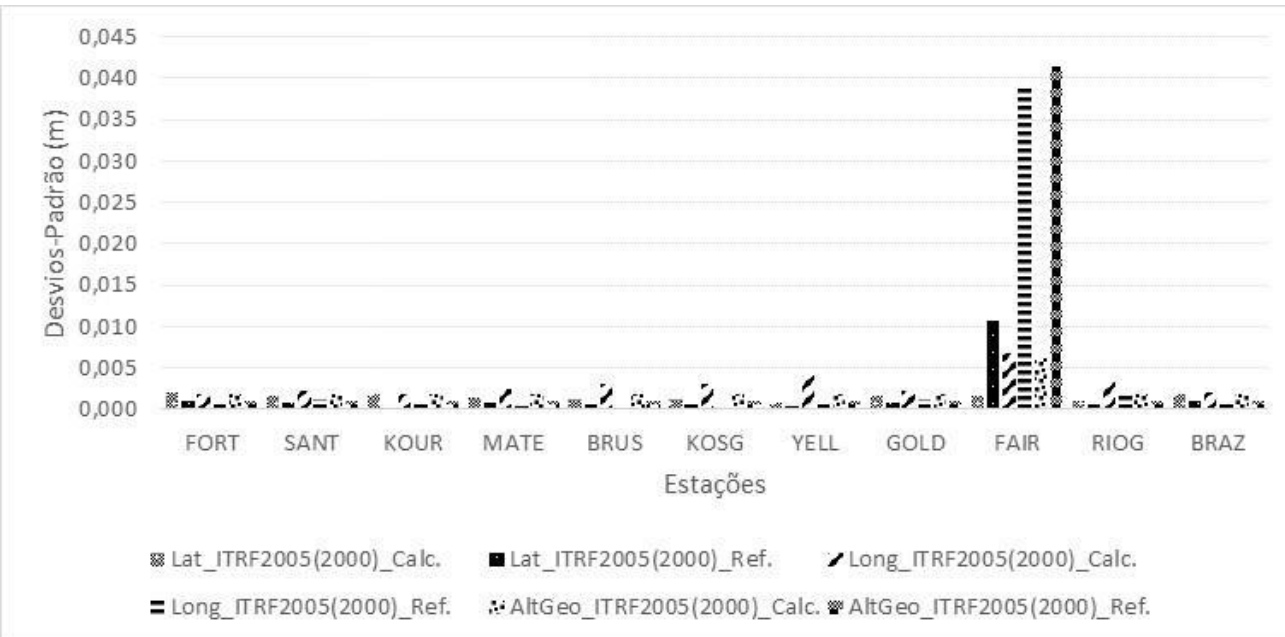

Figura 7: Desvios-padrão das coordenadas nos ITRF2005, época 2000,0.

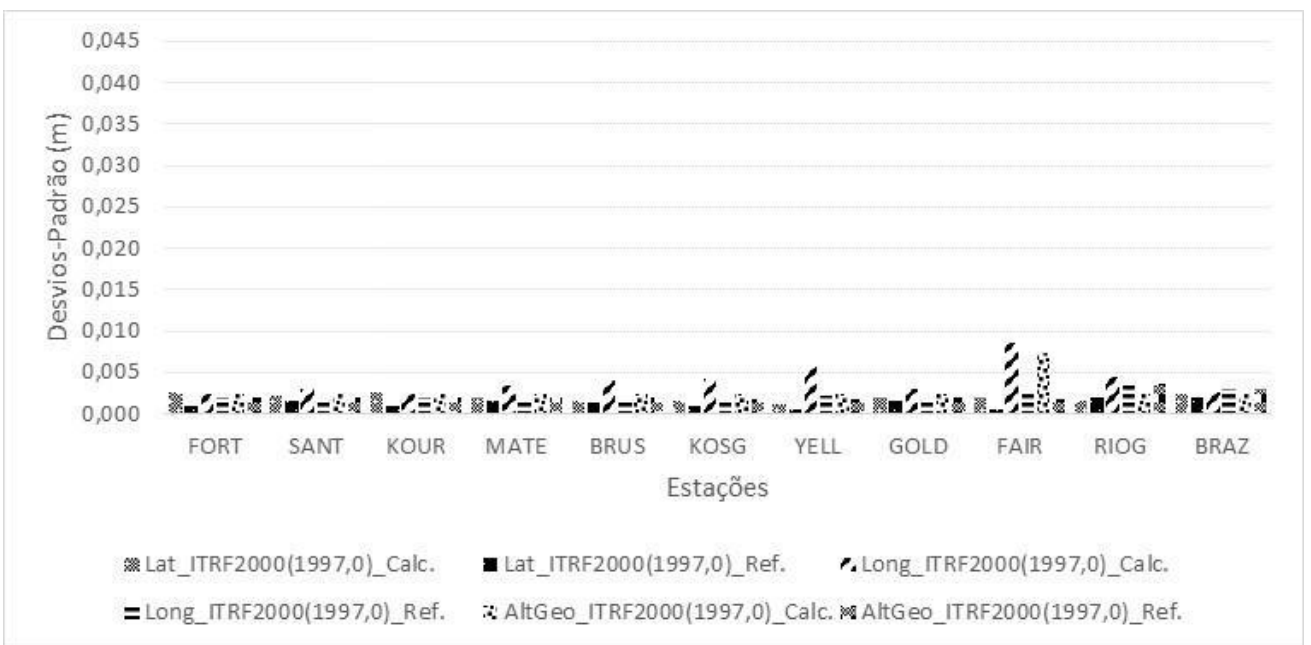

Figura 8: Desvios-padrão das coordenadas nos ITRF2000, época 1997,0.

Pode-se visualizar na Figura 7 que os desvios-padrão das coordenadas provenientes da solução ITRF2005, época 2000,0 para estação FAIR é centimétrico, enquanto nas demais estações na mesma solução é milimétrico. Porém, verifica-se que os desvios padrão das coordenadas no ITRF2005 dessa estação obtidos via propagação de variância foram menores que um centímetro, pois houve melhora na determinação posicional na realização ITRF2008, época 2005,0.

Na Figura 8 pode-se observar os desvios-padrão subcentimétricos para todas as estações, porém nota-se que a pior precisão foi encontrada na estação FAIR.

Pode-se observar que houve uma melhora sensível na determinação das coordenadas na realização ITRF2005, época 2005,0 em relação à solução ITRF2000, época 1997,0.

\section{Considerações finais e conclusões}


A transformação entre sistemas de referência das coordenadas e velocidades das estações empregadas bem como a determinação de suas precisões foram realizadas. Verificou-se a importância do conhecimento das velocidades bem como suas respectivas precisões para que seja considerado a variação espaço-temporal das estações e determinadas as precisões nas atualizações realizadas.

Detectou-se discrepâncias planimétricas que variaram do centímetro ao decímetro em cinco anos (2000 a 2005), quando não se atualizam as coordenadas. Os maiores valores de discrepância posicionais, com exceção da estação FAIR (Placa Norte-Americana), ocorreram nas estações MATE (Placa da Eurásia) e SANT (Sul-Americana) as quais estão localizadas em regiões próximas às bordas de placas tectônicas (convergentes). Embora a estação RIOG esteja localizada na placa Sul-Americana, a qual faz fronteira ao sul com a placa da Scotia (oceânica) e possuem movimentos transformantes, a mesma apresentou deslocamento planimétrico igual à da estação BRAZ localizada na placa Sul-Americana.

No estudo realizado não foi detectado discrepância das coordenadas obtidas por meio da realização das transformações diretas do ITRF2008 para os ITRF2005 e ITRF2000 em relação às transformações parciais de ITRF2008 para ITRF2005 e de ITRF2005 para ITRF2000. Na transformação direta não são disponibilizadas as precisões dos 14 parâmetros no site (http://itrf.ensg.ign.fr/doc_ITRF/Transfo-ITRF2008_ITRFs.txt), o que impossibilita, deste modo, a propagação de variância na transformação entre sistemas de referência.

É importante realizar a propagação de variância na atualização de coordenadas bem como na transformação entre realizações do ITRS, pois desse modo, as precisões das estações de controle poderão ser propagadas no de ajustamento na estimação das coordenadas das estações de interesse.

É necessário que os centros nacionais/internacionais, responsáveis por disponibilizarem os parâmetros de transformação, fornecem as precisões com que os parâmetros foram estimados, possibilitando a propagação de variância. Ressalta-se também a necessidade do conhecimento das precisões das coordenadas e das velocidades dos pontos.

\section{REFERÊNCIAS BIBLIOGRÁFICAS}

Altamimi, Z., Collilieux, X., and Métivier, L. "Analysis and results of ITRF2008" (IERS Technical Note; $\mathrm{n}^{\circ}$ 37). Frankfurt am Main: Verlag des Bundesamts für Kartographie und Geodäsie, p. 54. 2012. Accessed October 30, 2014: http://www.iers.org/TN37.

Drewes, Hermann., and Heidbach, Oliver. "The 2009 horizontal velocity field for South America and the Caribbean", in: Geodesy for Planet Earth: Proceedings of the 2009 IAG Symposium, Buenos Aires, Argentina, 31 August - 4 September 2009, (International Association of Geodesy Symposia; 136),Springer,p.657-664,doi:10.1007/978-3-642-20338-1_81.

Franco, Guido A. G., and Moreno, Raúl A.G. "Change from ITRF98 epoch 1988.0 to ITRF2000 epoch 2004.0 in MEXICO" (paper presented at the XXIII Fig. Congress. Munich, Germany. October 8-13, 2006). Acesso $30 \quad$ outubro, 2014. http://www.fig.net/pub/fig2006/papers/ts26/ts26_04_gonzalezfranco_gomezmoreno_0847.pdf

Haasdyk, Joel and Janssen, Volker, "The many paths to a common ground: A comparison of transformations between GDA94 and ITRF”, (paper presented at International Global Navigation 
Satellite Systems Society Symposium 2011, University of New South Wales, Sydney, NSW, Australia, November 2011).

IGS. “International GNSS Service”. Last modified September 03, 2014. http://acc.igs.org/

ITRF. "International Terrestrial Reference Frame". Last modified April, 2014. http://itrf.ensg.ign.fr/.

Pimenta, Alterni F., Zanetti, Maria A. Z., Dalazoana, Regiane, and Oliveira, Reginaldo. "ITRF2000 e sua Utilização". Boletim de Ciências Geodésicas. 8/1 (2002), p. 59-70. Accessed December 18, 2013. http://ojs.c3sl.ufpr.br/ojs/index.php/bcg/ article/viewFile/1414/1168.

Prol, Fabricio S., Marcato Junior, J., Nievinsk, Felipe G., Gomes, Ronaldo L., and Paranhos Filho, Antônio C. "Transformação entre referenciais e cálculo de velocidades através do aplicativo web TREVel”. Revista Brasileira de Cartografia. 66/3 (2014), p.569-579. Accessed November 28, 2014. http://www.lsie.unb.br/rbc/index.php/rbc/article/view/664

Petit, Gérard, and Luzum, Brian, eds."IERS conventions 2010" (IERS Technical Note n³6). Frankfurt am Main: Verlag des Bundesamts für Kartographie und Geodäsie, 2010. 179 pp. $\begin{array}{llll}\text { Accessed June } & 6013 .\end{array}$ http://www.iers.org/nn_11216/IERS/EN/Publications/TechnicalNotes/tn36.html.

Rebischung, Paul. "IGb08: an update on IGS08". 2012. Accessed October 23, 2014. http://igscb.jpl.nasa.gov/pipermail/igsmail/2012/007853.html.

Sánchez, L., Seemüller, W., Drewes, H., Mateo, L., González, G., da Silva, A., Pampillón, J., Martinez, W., Cioce, V., Cisneros, D., and Cimbaro, S."Long-Term Stability of the SIRGAS Reference Frame and Episodic Station Movements Caused by the Seismic Activity in the SIRGAS Region", in Reference Frames for Applications in Geosciences, International Association of Geodesy Symposia, eds. Altamimi, Z. and Collilieux, X. (Springer-Verlag Berlin Heidelberg, 2013): 153-161.

SIRGAS. "Sistema de Referência Geocêntrico para as Américas". Last modified February 27, 2015. http://www.sirgas.org/index.php?id=15.

Soler, Tomás, and Marshall, John, "Rigorous transformation of variance-covariance matrices of GPS-derived coordinates and velocities". GPS Solutions. 6 (2002):76-90. Accessed November 27 , 2014, doi: 10.1007/s10291-002-0019-1.

Soler, Tomás, and Snay, Richard A. "Transforming positions and velocities between the International Terrestrial Reference Frame of 2000 e North American datum of 1983," Journal of Surveying Engineering 130 (2004):49-45. Accessed November 27, 2014. doi: 10.1061/(ASCE) 0733-9453.

Weston, Neil D., and Soler, Tomás , "Rigorous geodetic positioning in the Americas" (paper presented at the $8^{\text {th }}$ FIG Regional Conference 2012 Surveying towards Sustainable Development, Montevideo, Uruguay, 26 - 29, November 2012). Accessed November 28, 2014. http://www.fig.net/pub/uruguay/techprog.htm.

Recebido em janeiro de 2015. Aceito em março de 2015. 